\title{
Quantum power: a Lorentz invariant approach to Hawking radiation
}

\author{
Michael R. R. Good ${ }^{1,2, a} \mathbb{D}$, Eric V. Linder ${ }^{2,3, b}$ \\ ${ }^{1}$ Physics Department, Nazarbayev University, Nur-Sultan, Kazakhstan \\ ${ }^{2}$ Energetic Cosmos Lab, Nazarbayev University, Nur-Sultan, Kazakhstan \\ ${ }^{3}$ Berkeley Center for Cosmological Physics and Berkeley Lab, University of California, Berkeley, CA, USA
}

Received: 31 January 2022 / Accepted: 24 February 2022 / Published online: 7 March 2022

(C) The Author(s) 2022

\begin{abstract}
Particle radiation from black holes has an observed emission power depending on the surface gravity $\kappa=c^{4} /(4 G M)$ as

$P_{\text {black hole }} \sim \frac{\hbar \kappa^{2}}{6 \pi c^{2}}=\frac{\hbar c^{6}}{96 \pi G^{2} M^{2}}$,

while both the radiation from accelerating particles and moving mirrors (accelerating boundaries) obey similar relativistic Larmor powers,

$P_{\text {electron }}=\frac{q^{2} \alpha^{2}}{6 \pi \epsilon_{0} c^{3}}, \quad P_{\text {mirror }}=\frac{\hbar \alpha^{2}}{6 \pi c^{2}}$,

where $\alpha$ is the Lorentz invariant proper acceleration. This equivalence between the Lorentz invariant powers suggests a close relation that could be used to understand black hole radiation. We show that an accelerating mirror with a prolonged metastable acceleration plateau can provide a unitary, thermal, energy-conserved analog model for black hole decay.
\end{abstract}

\section{Introduction}

The Equivalence Principle teaches us that gravitation is equivalent to a combination of acceleration and curvature, the last of these being unimportant on sufficiently small scales. Moreover we know that external effects on quantum fields creates particles, and this ties together black hole particle production, thermal baths observed by accelerating observers, and moving mirror acceleration radiation, e.g. the Hawking [1], Unruh [2], and Moore-DeWitt-Davies-Fulling [35] effects. However, we also know that constant acceleration is insufficient: e.g. consider the question of whether an electron sitting on a laboratory table in an eternal constant gravitational field of the Earth radiates (or the relationship

\footnotetext{
a e-mail: michael.good@nu.edu.kz (corresponding author)

be-mail: evlinder@lbl.gov
}

to the radiation from a mass freely-falling into a black hole [6-9]). Along the same lines, an eternal exactly uniformly accelerating boundary (moving mirror) evidently does not emit energy to an observer at infinity, e.g. [10]. There is not yet a consensus on the subtleties and non-intuitive behavior of eternal uniform acceleration (see [11] for a possible rationale of the distinction between chosen vacuum states).

Another aspect of great interest [12] is that asymptotically static mirrors preserve unitarity and information [13]. We explore a model that merges these two regimes of uniform acceleration and zero acceleration and show that this system, intuitively, can radiate particles for an extended time with constant power. The system will not only preserve information but emit thermal energy, conserve total radiated energy, and emit finite total particles, without infrared divergence. This model serves as an analog for complete black hole evaporation.

Related explorations are not without precedent. Black hole evaporation has close acceleration analogs [14] including moving mirror models $[4,15]$. Asymptotic infinite acceleration trajectories [16], like the accelerated boundary correspondences for the Schwarzschild, Reissner-Nordström, and Kerr black holes [17-19], evolve to eternal thermal equilibrium solutions [20]. Asymptotic finite acceleration (asymptotic uniform acceleration) correspond to extremal black holes [21-24] while asymptotic constant velocity (zero acceleration) can give information preserving quasi-thermal solutions describing black hole remnant models (e.g. [25-31]). Recently, particular emphasis is placed on unitary complete black hole evaporation models which are characterized by asymptotic zero-velocity mirrors (e.g. [32-38]).

Entanglement entropy [39], and hence information, is tied directly to the mirror trajectory [40]. However, the distant observer detects the radiated power, not the entropy. We investigate the connection between these for complete black hole evaporation via the analog case of uniform acceleration. 
Uniform acceleration mirrors are generally thought to emit zero energy $[41,42]$. In our case, we will explore metastable uniform acceleration, where there is an extended but finite period of constant power emission. We will confirm that the stress is zero during this plateau period but find that the power is not. We will discuss how this is a consequence of definitions, and in particular, of assuming asymptotic inertia in the derivation of quantum power. The model presented here will preserve information, evolve to thermal equilibrium, and conserve emitted energy, providing an analog for a black hole that completely evaporates away into radiation.

In Sect. 2 we exhibit the mirror dynamics of acceleration and velocity with the desired properties, leading in Sect. 3 to evolution with quantum purity (information preservation) from the finite entanglement entropy with a Page turnover. Section 4 computes the quantum Larmor power and total energy radiated, linking the mirror parameters with black hole properties. We conclude in Sect. 5, highlighting the unitarity and thermality of the analog model for black hole evaporation.

\section{Acceleration and velocity}

We seek a mirror acceleration that dies to zero at $\pm \infty$ (to preserve information) and has a constant plateau at some maximum acceleration (for metastable thermal power). We can arrange the maximum to be at time $t=0$, for example. We would also like to be able to adjust the duration of the plateau, to study the scaling. A simple model is

$\alpha=\alpha_{0} e^{-\left(t / t_{\star}\right)^{j}} \operatorname{sgn}(t)$.

The metastable plateau runs over $|t| \lesssim t_{\star}$; at $t=t_{\star}$ the acceleration falls to $1 / e$ of its maximum value $\alpha_{0}$. As a foreshadowing, we expect the power emission to determine the black hole lifetime, $\dot{M} \sim P \sim M^{-2}$, where $M$ is the black hole mass, so we anticipate a successful analog model will have $t_{\star} \sim M^{3}$.

We take $j$ to be a positive even integer so that $\alpha$ will die to zero for $t \rightarrow \pm \infty$. Large $j$ gives a flatter plateau and a steeper fall off to approach zero. For example, the acceleration plateau stays within a fraction $\epsilon$ of the maximum for $|t|<t_{\star} \epsilon^{1 / j}$ so for $j=4(8)$ it is within $1 \%$ of maximum out to $|t|<0.32 t_{\star}\left(0.56 t_{\star}\right)$. The limit $j \rightarrow \infty$ gives a box function for the plateau. This approaches equilibrium emission on the plateau. The sign flip (change in direction) in acceleration at $t=0$ is so the mirror comes back to rest (not merely inertial, but static) at future infinity. Since power depends on $\alpha^{2}$, the sign flip does not affect the power detected by a distant observer. (One can easily regularize the sign flip through use of a tanh transition without affecting the results.)

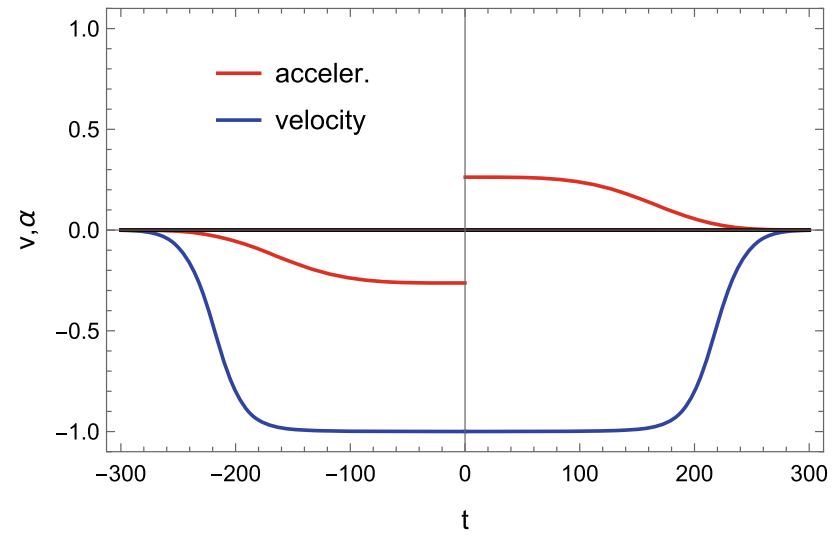

Fig. 1 The proper acceleration (red curve) of the mirror starts to the left (negative by convention), reaches a maximum magnitude (1/4M as $j \rightarrow \infty$ ), and has a sign (direction) change at $t=0$. The velocity (blue curve) of the $(1+1) \mathrm{D}$ mirror trajectory is always to the left (by convention); the mirror starts from zero speed, approaches the speed of light, then finally comes to rest. Here we plot for $M=1$ and $j=4$

The mirror velocity $v$ comes from the acceleration via the rapidity $\eta$, by $v=\tanh \eta$ and

$\sinh \eta(t) \equiv \int_{-\infty}^{t} d t^{\prime} \alpha\left(t^{\prime}\right)=\alpha_{0} t_{\star} \frac{\Gamma\left(1 / j,\left(t / t_{\star}\right)^{j}\right)}{j}$,

where $\Gamma$ is the incomplete Gamma function. The velocity smoothly goes from 0 to a maximum near the speed of light and back to 0 , without changing sign. The maximum velocity will be reached at $t=0$, where the incomplete Gamma function becomes a complete one, so

$$
\begin{aligned}
\sinh \eta(t=0) & =\alpha_{0} t_{\star} \frac{\Gamma(1 / j)}{j} \equiv Q \\
v_{\max } & =\left[1+Q^{-2}\right]^{-1 / 2} .
\end{aligned}
$$

When $j \rightarrow \infty$, then $Q=\alpha_{0} t_{\star}$. Note the maximum Lorentz boost factor $\gamma_{\max }=\left(1+Q^{2}\right)^{1 / 2}$. Figure 1 shows the acceleration and the resulting velocity.

\section{Entropy and unitarity}

Before proceeding further, let us establish this is a unitary analog model by observing that the entanglement entropy does not diverge, as expected for a mirror with asymptotic static end states [20]. From Eq. (2),

$S(t) \equiv \frac{\eta(t)}{6}=\frac{1}{6} \sinh ^{-1}\left(\alpha_{0} t_{\star} \frac{\Gamma\left(1 / j,\left(t / t_{\star}\right)^{j}\right)}{j}\right)$.

The entropy is asymptotically zero (no divergence), which signals purity. That is, in the limit $t \rightarrow \pm \infty, S \rightarrow 0$. This ensures that every field mode reflects to the observer. Without loss of field modes past a horizon, the model preserves quantum information during time evolution of the vacuum 


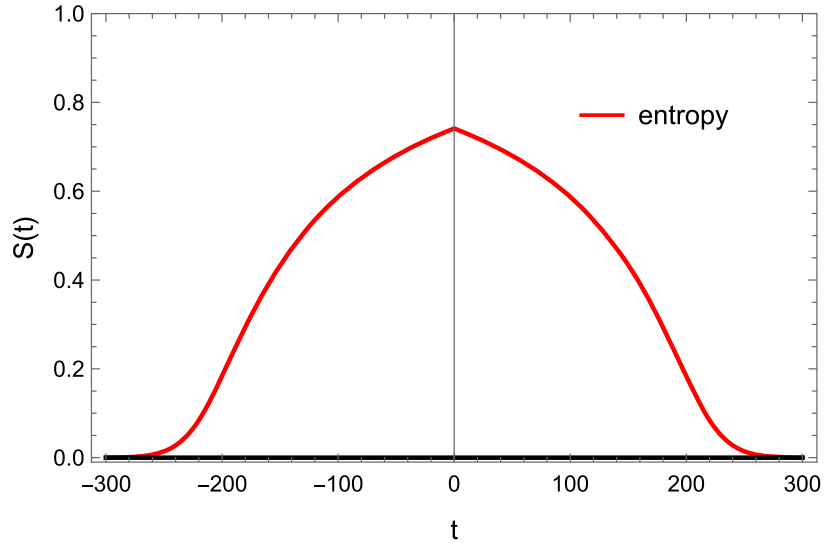

Fig. 2 Page curve turn-over of the von Neumann geometric entanglement entropy, Eq. (5), with asymptotic zeros and no divergence. This scalar measure of information demonstrates the model has no information loss by construction. Here $M=1$ and $j=4$ as in Fig. 1

state [43]. Figure 2 exhibits the expected Page curve turnover.

We can push this further, deriving thermodynamic entropy from entanglement entropy in the analog context. To reach the thermodynamic regime, we apply two equilibrium limits: flatness $j \rightarrow \infty$, and central time $t \rightarrow 0^{-}$. The value of the rapidity here is given by $\sinh \eta_{\star}=\alpha_{0} t_{*}=12 \pi M^{2}=$ $3 \pi /\left(4 \kappa^{2}\right)$ (as seen for $t_{*}$ in Sect. 4 in the $j \rightarrow \infty$ limit), with $\kappa$ the surface gravity. The entanglement is then

$S=\frac{2 \times 1}{6} \sinh ^{-1}\left(\alpha_{0} t_{\star}\right)=\frac{1}{3} \sinh ^{-1}\left(\frac{3 A}{4}\right)$.

In the first step, we have translated from dynamics to entanglement using the rapidity-entropy relation [44] and accounted for $(3+1)$ dimensions [45], where the additive modulus entanglement entropy is twice the one-sided entropy of a mirror in $(1+1)$ dimensions, $S=2(\eta / 6)$. In the second step, we have written the $(3+1)$ dimensional entanglement entropy in terms of the area of the analog black hole, $A=\pi / \kappa^{2}$, which illustrates thermodynamic entropy, $S=A / 4$, of the gravitational analog in the geometric limit $A \rightarrow 0$.

\section{Power and total energy}

The relativistic Larmor form for power, familiar from electrodynamics [46], also applies to the energy radiated from accelerating mirrors [45]. In the latter case

$P(t)=\frac{\hbar \alpha^{2}(t)}{6 \pi c^{2}}$,

where $\alpha$ is the frame-invariant proper acceleration. This measure is a good candidate for what the observer detects at asymptotic infinity. It is a Lorentz invariant corresponding

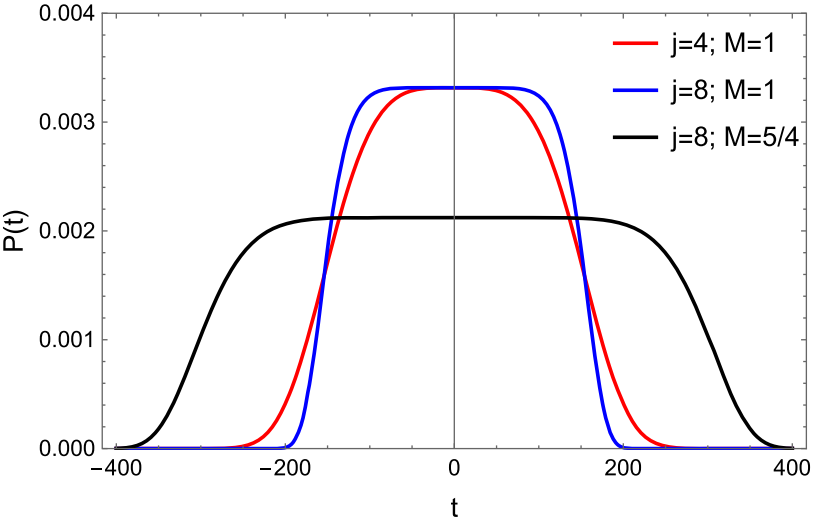

Fig. 3 The acceleration plateau results in a $(3+1) D$ power emission exhibiting an equilibrium emission plateau, with asymptotic finality (as $t \rightarrow \pm \infty$ ) corresponding to complete evaporation

to the emitted radiation from both sides of a $(1+1) \mathrm{D}$ moving mirror, as well as the emitted power for a $(3+1) \mathrm{D}$ moving mirror.

Thus the power for the corresponding $(3+1)$ dimensional situation of Eq. (1) is

$P(t)=\frac{\hbar \alpha_{0}^{2}}{6 \pi c^{2}} e^{-2\left(t / t_{\star}\right)^{j}}=\frac{c^{5}}{G} \frac{1}{6 \pi}\left(\frac{\alpha}{\alpha_{\mathrm{Pl}}}\right)^{2}$,

where here we explicitly show the "Planck power" $c^{5} / G$ and "Planck acceleration" $\alpha_{\mathrm{Pl}} \equiv c / t_{\mathrm{Pl}}$, although usually elsewhere we work in units where $\hbar=c=G=1$.

Figure 3 shows the emitted power $P(t)$ with its plateau, increasingly in equilibrium for large values of $j$, and vanishing at asymptotically early and late times. The asymptotically zero emission signals the end of evaporation, e.g. of the (ana$\log$ ) black hole, and a resulting finite total energy.

To find the total energy emitted by the evaporated $(3+1) \mathrm{D}$ black hole analog, one can integrate the power over coordinate time, $E=\int_{-\infty}^{+\infty} P(t) d t$. The result is

$E=\frac{\alpha_{0}^{2} t_{\star}}{3 \pi} \frac{\Gamma(1 / j)}{j 2^{1 / j}}$,

where now we have a complete Gamma function. As $j \rightarrow \infty$, the second fraction goes to 1 .

We can use this to fix $t_{\star}$ in terms of $E$ and $\alpha_{0}$. In particular, in the black hole context it is natural to take the correspondence that the total energy emitted is the mass $M$ of the black hole, and the acceleration during the equilibrium emission is the surface gravity, i.e. $\alpha_{0}=\kappa \equiv 1 /(4 M)$. This then implies that

$$
\begin{aligned}
t_{\star} & =\frac{3 \pi E}{\alpha_{0}^{2}} \frac{j 2^{1 / j}}{\Gamma(1 / j)} \\
& \rightarrow 48 \pi M^{3} \frac{j 2^{1 / j}}{\Gamma(1 / j)} \rightarrow 48 \pi M^{3}=\frac{3 \pi}{4 \kappa^{3}},
\end{aligned}
$$


The first right arrow takes the correspondence, and the second arrow takes the $j \rightarrow \infty$ limit. We see that as expected from the correspondence with black hole decay, the characteristic time scale $t_{\star} \sim M^{3}$. (In the mirror model, the radiation does not stay thermal beyond $t_{\star}$ so the total decay time is not defined for $j$ finite.) Note that under this correspondence, $Q=12 \pi M^{2}$ in Eq. (3). We used this expression in Sect. 3 to evaluate the thermodynamic entropy.

Conversely, we can write $\alpha_{0}^{2}=3 \pi E / t_{\star}$ in the limit and find

$P=\frac{c^{5}}{G} \frac{G M}{2\left(c t_{\star}\right) c^{2}}$,

i.e. there is a fundamental limit in that the decay time $t_{\star}$ must be long enough that the Planck power is not exceeded: one cannot radiate away the energy in shorter than a light crossing time.

This accelerating mirror model therefore provides an ana$\log$ to the concept that a physical black hole emits finite energy, that this energy is the total mass of the black hole itself for complete evaporation, the evaporation time $\sim M^{3}$, and the entropy $\sim A / 4$, consistent with surface gravity $\sim 1 /(4 M)$ in the thermodynamic limit.

\section{Conclusion}

We have presented an analog model using the dynamical Casimir effect for accelerating boundaries (mirrors) to describe a black hole that evaporates away completely. The approach uses the quantum power formula,

$P_{\text {mirror }}=\frac{\hbar \alpha^{2}}{6 \pi c^{2}}$,

where $\alpha$ is the Lorentz invariant proper acceleration. The quantum power approach works equivalently for (mirror) acceleration and (black hole) surface gravity. Since particle radiation from black holes has an observed emission power depending on the surface gravity $\kappa=c^{4} /(4 G M)$, as

$P_{\text {black hole }} \sim \frac{\hbar \kappa^{2}}{6 \pi c^{2}}=\frac{\hbar c^{6}}{96 \pi G^{2} M^{2}}$,

the connection between acceleration and gravity, inclusive with classical Larmor radiation from electrons,

$P_{\text {electron }}=\frac{q^{2} \alpha^{2}}{6 \pi \epsilon_{0} c^{3}}$,

is tied together from the underlying symmetry of Lorentz invariance.

The accelerating boundary correspondence exhibits the desirable characteristics of unitarity, thermality (equilibrium emission), and energy conservation. The finite entropy with Page turnover preserves information. The model thus describes a black hole that completely evaporates away in a physically reasonable manner. Other unique trajectories are tractable.

From a static state, the mirror accelerates to a velocity that can approach the speed of light (and the maximum rapidity is closely related to entropy, and black hole mass), before asymptotically becoming static again. While the specific model is of a mirror instantaneously reversing acceleration direction, a simple (e.g. tanh) regularization works in the same way.

The metastable plateau becomes flatter, more in equilibrium, as the superGaussian parameter $j$ increases. While the formal limit of equilibrium is $j \rightarrow \infty$, even for $j=4$ the plateau is flat to $1 \%$ for an extended period. In the limit there is a clear correspondence between the mirror acceleration and black hole surface gravity (and hence mass), total energy radiated and black hole total energy (mass), entropy and black hole area, and Larmor power and Hawking power.

While for a unitary model radiation episodes must occur with both positive and negative energy fluxes (and zero flux in the exact constant acceleration limit), the power always remains positive. The absence of $k_{B}$ in Eq. (13) agrees with use beyond the regime or limits of thermodynamic equilibrium. As a Lorentz invariant power is an interesting and mostly unstudied avenue by which to approach the correspondence with black holes.

A cautionary remark regarding quantum power, Eq. (13), is in order. Consider the apparent discrepancy between zero energy flux and the non-zero quantum power during metastable uniform acceleration. This is a result of key differences in definitions between energy flux and quantum power. For instance, the quantum power formula is not applicable for eternal uniform acceleration, but is perfectly applicable for metastable uniform acceleration (as long as the global trajectory is asymptotically inertial). The reason for this is that asymptotic inertia is an assumption required to obtain the quantum power formula itself [45].

Asymptotic inertial radiated power is not an inconsequential subtlety. It is related to the long standing debate $[47,48]$ over whether a uniformly accelerated point charge radiates. Since the quantum power formula does not apply to globallydefined eternal uniform acceleration, our results do not conflict with the confirmations that a globally uniformly accelerated mirror does not radiate energy [41] but does radiate particles (as is well-established [49-51]). Nor does it conflict with the fact that uniformly accelerated point-like structureless sources emit only zero-energy Rindler particles [52].

Future work on the model presented here could explore the approach to equilibrium and the development of the particle spectrum. Conventional computation of the beta Bogolyubov coefficients is not tractable here, but the asymptotic static states guarantee there will be no infrared divergence (no black hole remnant) and a finite total number of particles emitted. Alternative attempts at the spectrum may yield insights into 
the particle production and other physics of black hole radiation.

Acknowledgements Funding from the FY2021-SGP-1-STMM Faculty Development Competitive Research Grant No. 021220FD3951 at Nazarbayev University is acknowledged. This work is supported in part by the Energetic Cosmos Laboratory. EL is supported in part by the U.S. Department of Energy, Office of Science, Office of High Energy Physics, under contract no. DE-AC02-05CH11231.

Data Availability Statement This manuscript has no associated data or the data will not be deposited. [Authors' comment: All data generated in this study is contained in the article.]

Open Access This article is licensed under a Creative Commons Attribution 4.0 International License, which permits use, sharing, adaptation, distribution and reproduction in any medium or format, as long as you give appropriate credit to the original author(s) and the source, provide a link to the Creative Commons licence, and indicate if changes were made. The images or other third party material in this article are included in the article's Creative Commons licence, unless indicated otherwise in a credit line to the material. If material is not included in the article's Creative Commons licence and your intended use is not permitted by statutory regulation or exceeds the permitted use, you will need to obtain permission directly from the copyright holder. To view a copy of this licence, visit http://creativecomm ons.org/licenses/by/4.0/.

Funded by $\mathrm{SCOAP}^{3}$.

\section{References}

1. S. Hawking, Commun. Math. Phys. 43, 199 (1975). https://doi.org/ 10.1007/BF02345020

2. W.G. Unruh, Phys. Rev. D 14, 870 (1976). https://doi.org/10.1103/ PhysRevD.14.870

3. G.T. Moore, J. Math. Phys. 11, 2679 (1970). https://doi.org/10. 1063/1.1665432

4. B.S. DeWitt, Phys. Rep. 19, 295 (1975). https://doi.org/10.1016/ 0370-1573(75)90051-4

5. S.A. Fulling, P.C.W. Davies, Proc. R. Soc. Lond. A Math. Phys. Sci. 348, 393 (1976). https://doi.org/10.1098/rspa.1976.0045

6. F. Rohrlich, Il Nuovo Cimento 21, 811 (1961). https://doi.org/10. 1007/BF02785607

7. F. Rohrlich, Ann. Phys. 22, 169 (1963). https://doi.org/10.1016/ 0003-4916(63)90051-4

8. M. Pauri, M. Vallisneri, Found. Phys. 29, 1499 (1999). https://doi. org/10.1023/A:1018821619763 arXiv:gr-qc/9903052

9. M.O. Scully, S. Fulling, D.M. Lee, D.N. Page, W.P. Schleich, A.A. Svidzinsky, Proc. Natl. Acad. Sci. 115, 8131 (2018). https://doi. org/10.1073/pnas.1807703115

10. L. Ford, A. Vilenkin, Phys. Rev. D 25, 2569 (1982). https://doi. org/10.1103/PhysRevD.25.2569

11. N. Nicolaevici, Class. Quantum Gravity 32, 045013 (2015). https:// doi.org/10.1088/0264-9381/32/4/045013. arXiv:1501.00119 [hep-th]

12. P. Chen, G. Mourou, Phys. Rev. Lett. 118, 045001 (2017). https:// doi.org/10.1103/PhysRevLett.118.045001 arXiv:1512.04064 [grqc]

13. P. Chen, D.-H. Yeom, Phys. Rev. D 96, 025016 (2017). https://doi. org/10.1103/PhysRevD.96.025016 arXiv:1704.08613 [hep-th]

14. P.C.W. Davies, J. Phys. A 8, 609 (1975). https://doi.org/10.1088/ $0305-4470 / 8 / 4 / 022$
15. P. Davies, S. Fulling, Proc. R. Soc. Lond. A Math. Phys. Sci. A356, 237 (1977). https://doi.org/10.1098/rspa.1977.0130

16. R.D. Carlitz, R.S. Willey, Phys. Rev. D 36, 2327 (1987). https:// doi.org/10.1103/PhysRevD.36.2327

17. M.R.R. Good, P.R. Anderson, C.R. Evans, Phys. Rev. D 94, 065010 (2016). https://doi.org/10.1103/PhysRevD.94.065010 arXiv: 1605.06635 [gr-qc]

18. M.R.R. Good, Y.C. Ong, Eur. Phys. J. C 80, 1169 (2020). https:// doi.org/10.1140/epjc/s10052-020-08761-7 arXiv:2004.03916 [gr-qc]

19. M.R.R. Good, J. Foo, E.V. Linder, Class. Quantum Gravity 38, 085011 (2021). https://doi.org/10.1088/1361-6382/abebb6. arXiv:2006.01349 [gr-qc]

20. I.A. Reyes, Phys. Rev. Lett. 127, 051602 (2021). https://doi.org/ 10.1103/PhysRevLett.127.051602 arXiv:2103.01230 [hep-th]

21. S. Liberati, T. Rothman, S. Sonego, Phys. Rev. D 62, 024005 (2000). https://doi.org/10.1103/PhysRevD.62.024005 arXiv:gr-qc/0002019

22. T. Rothman, Phys. Lett. A 273, 303 (2000). https://doi.org/10. 1016/S0375-9601(00)00515-6 arXiv:gr-qc/0006036

23. M.R.R. Good, Phys. Rev. D 101, 104050 (2020). https://doi.org/ 10.1103/PhysRevD.101.104050 arXiv:2003.07016 [gr-qc]

24. J. Foo, M.R.R. Good, JCAP 01, 019 (2021). https://doi.org/10. 1088/1475-7516/2021/01/019 arXiv:2006.09681 [gr-qc]

25. P. Chen, Y.C. Ong, D.-H. Yeom, Phys. Rep. 603, 1 (2015). arXiv:1412.8366 [gr-qc]

26. M.R.R. Good, Y.C. Ong, JHEP 07, 145 (2015). https://doi.org/10. 1007/JHEP07(2015)145 arXiv:1506.08072 [gr-qc]

27. M.R.R. Good, K. Yelshibekov, Y.C. Ong, JHEP 03, 013 (2017). https://doi.org/10.1007/JHEP03(2017)013 arXiv:1611.00809 [grqc]

28. M.R.R. Good, Reflecting at the Speed of Light (World Scientific, Singapore, 2017). arXiv:1612.02459

29. M.R. Good, Universe 4, 122 (2018). https://doi.org/10.3390/ universe 4110122

30. A. Myrzakul, M.R. Good, in 15th Marcel Grossmann Meeting on Recent Developments in Theoretical and Experimental General Relativity, Astrophysics, and Relativistic Field Theories (2018). arXiv:1807.10627 [gr-qc]

31. M.R. Good, Y.C. Ong, A. Myrzakul, K. Yelshibekov, Gen. Relativ. Gravit. 51, 92 (2019). https://doi.org/10.1007/ s10714-019-2575-5. arXiv:1801.08020 [gr-qc]

32. W.R. Walker, P.C.W. Davies, J. Phys. A Math. Gen. 15, L477 (1982). https://doi.org/10.1088/0305-4470/15/9/008

33. M.R. Good, E.V. Linder, Phys. Rev. D 97, 065006 (2018). https:// doi.org/10.1103/PhysRevD.97.065006 arXiv:1711.09922 [gr-qc]

34. M.R. Good, E.V. Linder, Phys. Rev. D 99, 025009 (2019). https:// doi.org/10.1103/PhysRevD.99.025009 arXiv:1807.08632 [gr-qc]

35. M.R. Good, E.V. Linder, F. Wilczek, Phys. Rev. D 101, 025012 (2020). https://doi.org/10.1103/PhysRevD.101.025012 arXiv: 1909.01129 [gr-qc]

36. M.R.R. Good, P.R. Anderson, C.R. Evans, Phys. Rev. D 88, 025023 (2013). https://doi.org/10.1103/PhysRevD.88.025023 arXiv:1303.6756 [gr-qc]

37. M.R.R. Good, E.V. Linder, Phys. Rev. D 96, 125010 (2017). https:// doi.org/10.1103/PhysRevD.96.125010 arXiv:1707.03670 [gr-qc]

38. M.R.R. Good, E.V. Linder, F. Wilczek, Mod. Phys. Lett. A 35, 2040006 (2020). https://doi.org/10.1142/S0217732320400064 arXiv:2108.11188 [quant-ph]

39. C. Holzhey, F. Larsen, F. Wilczek, Nucl. Phys. B 424, 443 (1994). https://doi.org/10.1016/0550-3213(94)90402-2 arXiv:hep-th/9403108

40. I. Akal, Y. Kusuki, N. Shiba, T. Takayanagi, Z. Wei, Phys. Rev. Lett. 126, 061604 (2021). https://doi.org/10.1103/PhysRevLett. 126.061604 arXiv:2011.12005 [hep-th] 
41. N. Birrell, P. Davies, Quantum Fields in Curved Space, Cambridge Monographs on Mathematical Physics (Cambridge Univ. Press, Cambridge, 1984). https://doi.org/10.1017/CBO9780511622632

42. M.R.R. Good, E.V. Linder, (2021). arXiv:2108.07451 [gr-qc]

43. F. Wilczek, in International Symposium on Black holes, Membranes, Wormholes and Superstrings (1993), pp. 1-21. arXiv:hep-th/9302096

44. M.R.R. Good, Y.C. Ong, J. High Energy Phys. 1507, 145 (2015). https://doi.org/10.1007/JHEP07(2015)145 arXiv:1506.08072 [grqc]

45. A. Zhakenuly, M. Temirkhan, M. Good, P. Chen, Symmetry 13, 653 (2021). https://doi.org/10.3390/sym13040653 arXiv:2101.02511 [gr-qc]

46. J.D. Jackson, Classical electrodynamics, 3rd edn. (Wiley, New York, 1999)

47. T. Fulton, F. Rohrlich, Ann. Phys. 9, 499 (1960). https://doi.org/
10.1016/0003-4916(60)90105-6

48. D.G. Boulware, Ann. Phys. 124, 169 (1980). https://doi.org/10. 1016/0003-4916(80)90360-7

49. B.S. Kay, U. Lupo, Class. Quantum Gravity 33, 215001 (2016). $\quad$ https://doi.org/10.1088/0264-9381/33/21/215001. arXiv: 1502.06582 [gr-qc]

50. N. Obadia, R. Parentani, Phys. Rev. D 67, 024021 (2003). https:// doi.org/10.1103/PhysRevD.67.024021 arXiv:gr-qc/0208019

51. N. Obadia, R. Parentani, Phys. Rev. D 67, 024022 (2003). https:// doi.org/10.1103/PhysRevD.67.024022 arXiv:gr-qc/0209057

52. G. Cozzella, S.A. Fulling, A.G.S. Landulfo, G.E.A. Matsas, Phys. Rev. D 102, 105016 (2020). https://doi.org/10.1103/PhysRevD. 102.105016 arXiv:2009.13246 [hep-th] 